\title{
Structural Strength Design and Navigation Capability Analysis of Polar Cruise
}

\author{
Zhang Xuchen ${ }^{1, *}$, Chi Linru ${ }^{1}$ and Li Ziqiang ${ }^{1}$ \\ ${ }^{1}$ School energy and power engineering, Wuhan university of technology, Wuhan, Hubei, 430063, China
}

\begin{abstract}
The "Ice Silk Road" is an effective extension of China's "One Belt, One Road" initiative. The construction of the "Ice Silk Road" and the development and utilization of polar waterways urgently need the equipment support of polar ships. Nowadays, the design and construction of polar cruise ships has gradually become a hot spot, but there is still a lot of room for development of such ships. Based on the relevant requirements of BV classification society and IACS, this article analyzes the current situation of polar cruises' structural strength and operational capabilities, and evaluates the overall capabilities of polar cruises, which has a certain reference value for the design and construction of cruise ships that will be developed in the future.
\end{abstract}

\section{Introduction}

In recent years, the global climate has continued to warm, and the development of polar resources and the continuous expansion of bipolar routes have gradually become a hot spot. Following this, the polar sailing ship industry ushered in a major development opportunity. However, opportunities are often accompanied by challenges. Compared with conventional ships sailing at sea, ships sailing in polar regions require additional strengthening of their hull structure due to the large ice load in the polar regions. At the same time, cruise ships need to traverse harsh seas and ice when sailing in the north and south poles. In addition to ensuring the comfort of passengers, the safety and stability of the cruise ship's power system is the key that cannot be ignored.

China Merchants Port Group is the largest public terminal operator in China. According to Wang Cuijun, Deputy General Manager of China Merchants Group, China Merchants Group has been studying the development situation of the cruise industry at home and abroad since 2013, and proposed to rely on the group's shipbuilding, ports, shipping, With the advantages of the entire cruise industry chain such as logistics and finance, "cruise construction" is the strategic development direction of the group. The "No. 1 Cruise Ship" named and delivered today and the cruise base that was solemnly laid are the important achievements and core measures of China Merchants' overall industrial chain layout of the cruise industry.

This paper selects a PC6 class expedition cruise ship classified as $\mathrm{BV}$ as the research object. According to the requirements of the $\mathrm{BV}$ specification and IACS PC specification, the software Mars developed by BV is used to conduct a normative check on the structure of the ship; at the same time, the inlet velocity and the inlet ice content of the two-phase flow through the horizontal tube are discussed in combination with the experiment and FLUENT simulation. The influence of the sea ice concentration field and the velocity field reveals the influence of the two-phase flow inlet parameters on the flow characteristics of the two-phase flow in the pipeline.

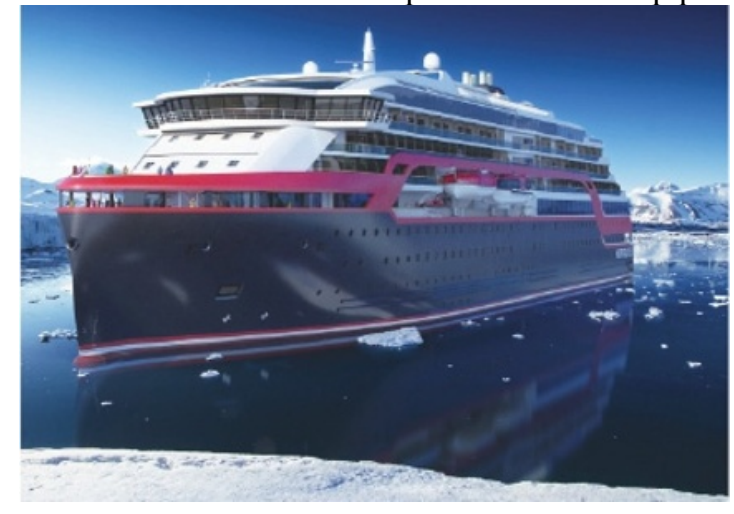

Fig. 1. Polar Cruise Pictures

\section{Design features required for Polar Cruises}

As an important platform for polar scientific research, polar ships have three key points that are different from conventional ship designs in terms of polar ship design:

\subsection{Combines ice dynamic characteristics}

In most cases, ships sailing in polar ice regions are in a mixed environment of ice and water. At this time, the external force acting on the hull is called ice hydrodynamics. This force includes both the force between the ship and the ice and the hydrodynamic force. Aspects. In the course of navigation and maneuvering in

* Corresponding author: zxc20000802@whut.edu.cn 
the ice region, the external force of the ship itself is more affected by the force of ice. General polar sailing ships should focus on the main engine power and ice breaking resistance when sailing in the ice zone. At the same time, they also need to have good hydrodynamic performance in an ice-free state. Ships should pay attention to the ability to pass in extreme ice areas, that is, when the ship is sailing at the minimum stable speed and the ship's power plant emits full power, it can still pass the maximum thickness of dense ice smoothly. Secondly, the speed of navigation in the ice region is also very important. The maximum speed that the ship's main engine can reach under specific ice conditions under a certain power. Generally, polar ships use dual-shaft and multi-propeller devices with more than two shafts, which have good maneuverability and can realize mobile operations in various directions. The driving force is the power propulsion of diesel engines, and the rudder and propellers have proper protection and structural reinforcement.

\subsection{Higher structural strength requirements}

In an icy environment, when a ship is sailing, its hull will generate an interaction force with sea ice, and there will be more additional loads on the part of the bow that is squeezed by the sea ice. At the same time, propellers and underwater thrusters, etc. Parts with weaker structural strength are also susceptible to the impact of thick ice and cause equipment failure. These are factors that have not been considered in the design and construction of conventional ships. Polar ships will also be subjected to the combined effects of various ice loads in lowtemperature environments, including bow impact ice load, bow flushing force, local ice pressure on the hull, and additional compression on the side during narrow channel passage or turning operations. Force, ice floe impact, etc. Due to the continuous squeezing of the ice layer in the bow, the force is concentrated, so in the design of the ship, the hulls of polar ships are mostly shorter in the longitudinal direction and wider in the transverse direction, thereby reducing the extra load generated when squeezing with sea ice.

\subsection{More weather risk factors}

Compared with the meteorological conditions and sea conditions in the middle and low latitude sea areas, the extremely low temperature in the polar regions will cause ice accumulation on the open-air equipment, and the escape routes are also easy to freeze; the widespread sea ice on the sea will cause underwater equipment and ice conditions The high latitude area has few base stations, which will affect the communication between ships and land, which is not conducive to obtaining navigation information in time; long-term polar nights will affect the line of sight. These more severe weather conditions require polar ships to have good seaworthiness and cold resistance. In the polar scientific research environment, the temperature is generally lower than $-30^{\circ} \mathrm{C}$. Therefore, when designing and constructing polar ships, the most important consideration is the cold-proof design of the ship. Each cabin and each system of the ship needs to be specially designed for cold protection and apply for related additional signs (polar cold protection mark/deicing cold protection mark). In addition, ship deck machinery and other open-air equipment should also have good cold resistance. When sailing in polar regions, various machinery and equipment can still operate and operate normally under low temperature environments.

\section{Strength analysis of hull structure}

\subsection{Strengthened ice structure}

\subsubsection{Strengthened area of ice structure}

For ships sailing in polar regions, the probability of collision with ice in different areas of the hull will be different. Therefore, the evaluation of the structural strength of the hull structure also needs to divide the hull into different areas for separate verification. According to the requirements of the BV code NR527 and the IACS PC code, the ship is mainly divided into 4 areas along the length of the ship: the bow area, the bow transition area, the midship area and the stern area. Each area is further divided into 3 areas in the vertical direction. The area includes ice zone, lower area, and bottom area.

\subsubsection{Local load on ice}

The consideration of ice load mainly divides the hull into two areas longitudinally: the bow area and the non-bow area. Assuming that the ice load is the average pressure Pavg acting on a rectangular block with the size of $\mathrm{Wxb}$, the design load plate aspect ratio $(\mathrm{w} / \mathrm{b})$ of the non-forehead area is set to 3.6, and the concentration force FNonBow and linear load of each partition are calculated to determine Load plate parameters.

For the pressure calculation of the bow area, the area needs to be further divided into 4 sub-areas. At the center of each sub-area, the ship length, displacement, and hull shape (waterline angle, longitudinal section angle, rib angle, and vertical direction of the frame) are considered. Angle etc.). After the constants corresponding to each ice level, the concentrated force F, line load Q, surface pressure $\mathrm{P}$ and the aspect ratio AR of the loaded plate are calculated to determine the design load plate parameters $b$, w, Pavg.

\subsubsection{Extra total strength load in ice area}

For ships sailing in polar regions, if the bow is not bulbous or vertical, the additional vertical ice load bending moment and shear force generated by the bow ice load need to be considered. The additional ice load bending moment When checking the structural strength with shear force, only the hydrostatic bending moment and shear force superimposed with the hull itself are considered, and the allowable standards for structural strength are also different. 


\subsection{Layout and calculation of the middle cross section}

\subsubsection{Layout of the middle cross section}

For ships sailing in general ice areas, the structural form of ice reinforcement is the horizontal frame type. The advantage of the horizontal frame type is that the utilization rate of the bones is higher. Compared with the longitudinal frame type, the material is saved, but the overall strength may be different. Relatively disadvantaged. The design of the mid-transverse section of the ship is that the side ice zone and the lower area are of the transverse frame type. The other outer plating areas including the other areas of the side and the bottom are all longitudinal frames, and all deck areas are also longitudinal frames.

\subsubsection{Middle cross section modeling}

In the initial verification process, there is no finite element analysis result, and it is difficult to define the contribution of the superstructure to the total strength. Under this circumstance, the participation of the building on the total strength will be defined as $50 \%$ and $100 \%$ respectively. The participation of the building is generally between $50 \%$ and $100 \%$. When it is defined as $50 \%$, it is to check the entire section. Whether the modulus of is sufficient, the check of total strength is conservative, and the total longitudinal stress of the upper building is the largest when it is defined as $100 \%$, in order to check the local strength of the upper building under the total longitudinal stress. All cases have been conservatively considered, and the problem of no finite element structure reference in the early stage has been solved. In the later stage, the finite element results can be used to verify.
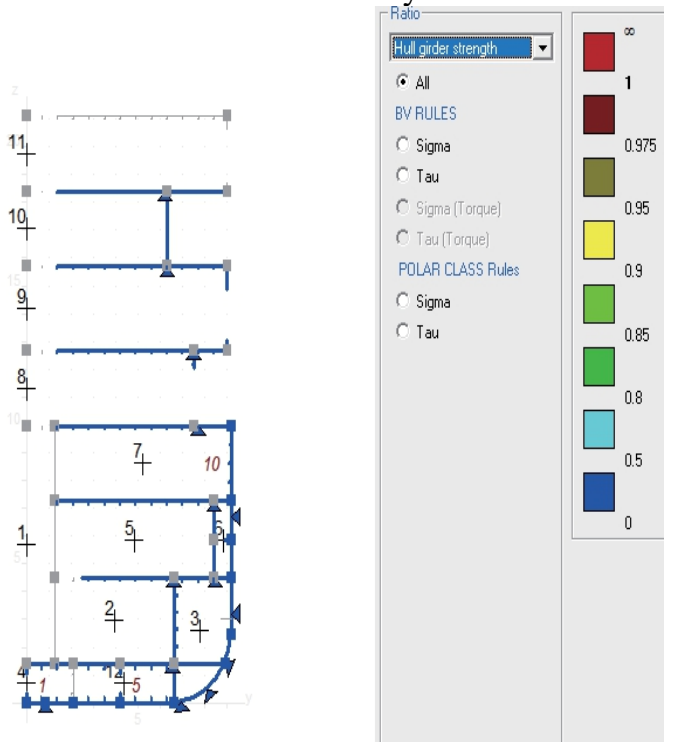

Fig. 2. Consider the result of building $50 \%$ participation in the total intensity

\subsubsection{Normative check result of middle cross section}

The current total strength is basically very rich. The existing structural strengthening of the ice area is relatively sufficient. The Shangiian has sufficient strength after considering $100 \%$ participation in the total strength. The specific results are shown in Figures 3 and 4.
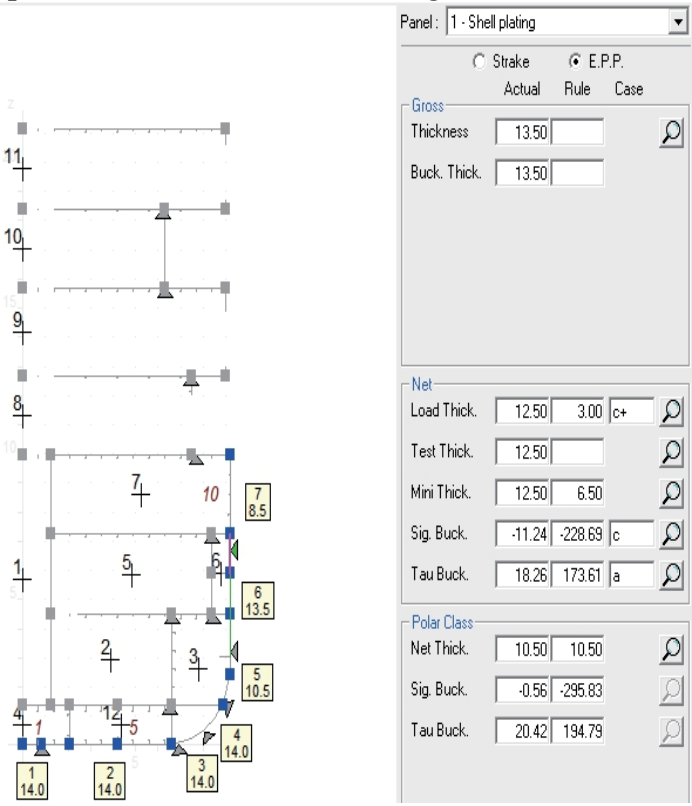

Fig. 3. Check results of the outer board in the ice zone
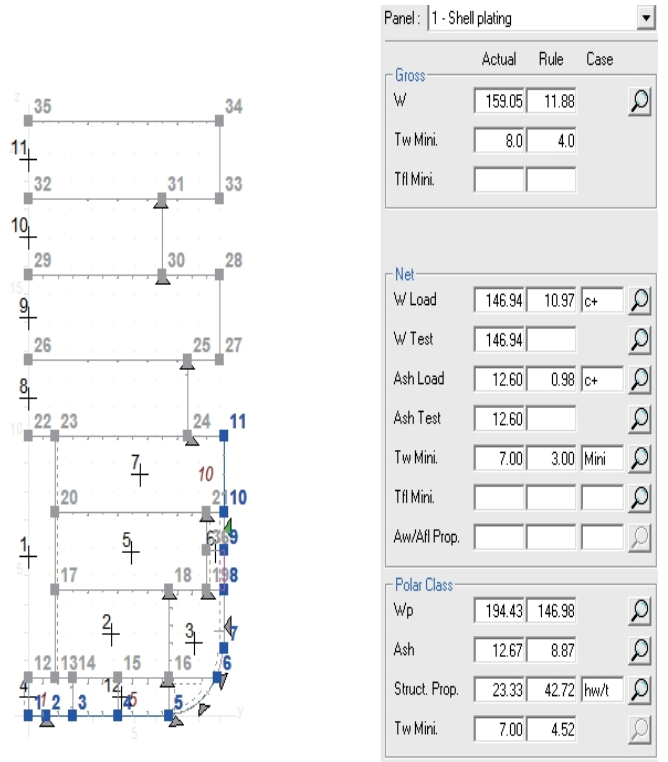

Fig. 4. Rib check results in the ice zone

\section{Analysis of two-phase flow in the piping system of a polar cruise ship during navigation}

\subsection{Experimental theory}

Set the sea ice particles to be smooth and inelastic, adopt the Euler-Euler model [6], treat the ice crystal particles and sea water as a continuous medium, and describe the flow characteristics of the two-phase flow by establishing the mass, momentum and energy conservation control equations of the continuous medium. 


\subsection{Analysis of flow characteristics of two-phase flow}

Experiments have found that moving beds of sea ice often occur when the inlet velocity of the two-phase flow is low. It appears that the top of the pipeline is a moving bed, and the bottom is an uneven flowing layer. In this case, a large number of ice crystal particles will accumulate in the upper part of the pipeline, increasing the risk of ice blockage. After repeated observations, at an ice content of $10 \%$, the critical inlet velocity for the occurrence of a moving sea bed is $1.0 \mathrm{~m} / \mathrm{s}$.
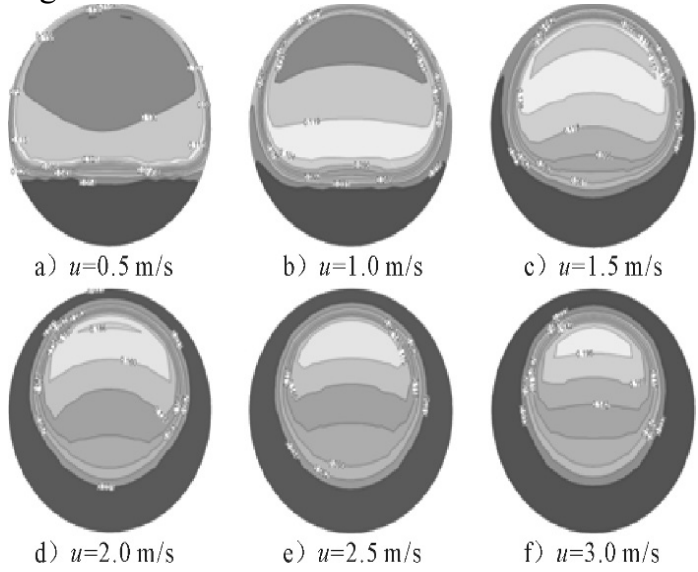

Fig. 5. Concentration distribution of ice crystals at different inlet flow rates

The possibility of the occurrence of a moving bed of sea ice in the cruise seawater cooling piping system will increase with the increase of the ice content of the twophase orifice and the decrease of the inlet flow rate. The appearance of the moving bed will also cause the stratification of the flow velocity in the pipe. With the increase of the ice content, the maximum flow velocity of the two-phase flow will deviate from the central axis of the pipe and move downwards. With the increase of the inlet flow velocity, the maximum velocity gradually moves from the central axis. The lower part returns to the position that coincides with the central axis, and the sea ice is distributed almost symmetrically along the circumference of the pipeline.
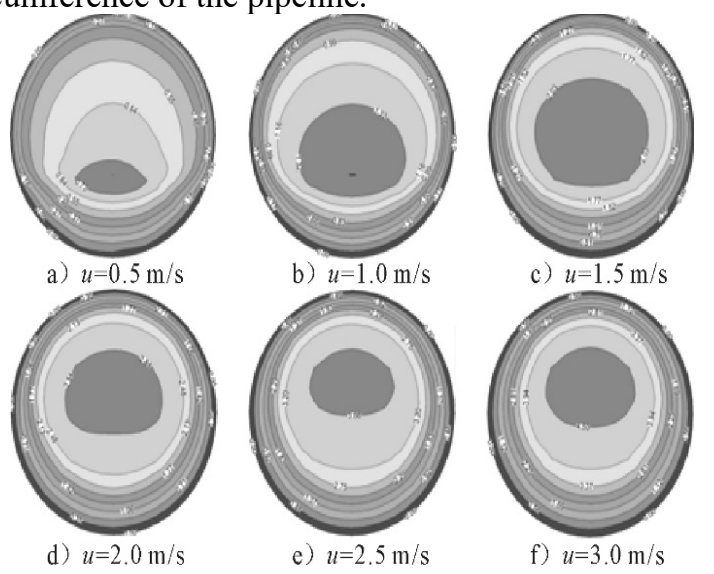

Fig. 6. Sea ice velocity distribution at different inlet velocities

The comparison of simulation cloud image analysis and experimental phenomena shows that the Euler-Euler model coupled mass transfer model is feasible to analyze the flow characteristics of two-phase flow. Using this method to simulate the change characteristics of the twophase flow field can reduce the cost of model experiments and provide theoretical support for the optimal design of the piping system.

\section{Conclusion}

At present, polar cruises still have great potential for development. There are many design preconditions and design requirements in terms of hull structural strength, especially in the area of ice structure strengthening. It is necessary to pay attention to the angle analysis of different areas of the hull colliding with ice; at the same time, polar cruises are in the process of sailing. The occurrence of ice blockage in the water cooling pipeline system is closely related to the two-phase flow inlet flow rate and the inlet ice rate. Therefore, it is necessary to provide a good reference for the preliminary work of the related design in the future and the later navigation process during the design.

\section{References}

1. Wang Jianwei, Duan Qinglin, Ji Shunying. Research progress of on-site measurement and inversion methods of ship structure ice load during ice navigation[J]. Advances in Mechanics, 2020, 50(00): 93-123.

2. Engineering-Marine Science and Engineering; Researchers from Harbin Engineering University Detail Research in Marine Science and Engineering (Numerical Simulation of a Polar Ship Moving in Level Ice Based on a One-Way Coupling Method)[J]. Journal of Mathematics, 2020.

3. Wang Zhipeng, Hao Zhailiu, Tian Yukui, Wu Baoshan Research on ice resistance prediction of polar transport ships based on FEM/SPH coupling method[J]. Ship Mechanics, 2021, 25(01): 9-15.

4. Geng Tuo, Zhao Zhongqiu, Xu Jing, Hao Jinfeng. Direct calculation of ice load and structural strength evaluation of polar ship[J]. Ship Engineering, 2019, 41(07): 1-4+104.

5. Han Duanfeng, Wang Yongkui, Ju Lei, Wang Qing. Phase field simulation of ice crystal growth during seawater freezing $[\mathrm{J}]$. Journal of Harbin Engineering University, 2020, 41(01): 1-8.

6. Jin Qiang, Zhang Jianing, Ge Yuan, Gao Bo. Ship ice resistance based on discrete element method in polar floating ice area[J]. Ship Engineering, 2020, 42(01): $35-41$. 